\title{
STOCHASTIC MODELING AIDS EPIDEMIOLOGY AND HIV PATHOGENESIS
}


This page is intentionally left blank 


\title{
STOCHASTIC MODELING OF
} AIDS EPIDEMIOLOGY AND HIV PATHOGENESIS

\author{
Tan Wai-yuan \\ Department of Mathematical Science \\ University of Memphis \\ Memphis, USA
}




\section{Published by}

World Scientific Publishing Co. Pte. Ltd.

P O Box 128, Farrer Road, Singapore 912805

USA office: Suite 1B, 1060 Main Street, River Edge, NJ 07661

UK office: 57 Shelton Street, Covent Garden, London WC2H 9HE

\section{British Library Cataloguing-in-Publication Data}

A catalogue record for this book is available from the British Library.

\section{STOCHASTIC MODELING OF AIDS EPIDEMIOLOGY AND HIV PATHOGENESIS}

Copyright $\odot 2000$ by World Scientific Publishing Co. Pte. Ltd.

All rights reserved. This book, or parts thereof, may not be reproduced in any form or by any means, electronic or mechanical, including photocopying, recording or any information storage and retrieval system now known or to be invented, without written permission from the Publisher.

For photocopying of material in this volume, please pay a copying fee through the Copyright Clearance Center, Inc., 222 Rosewood Drive, Danvers, MA 01923, USA. In this case permission to photocopy is not required from the publisher.

ISBN $981-02-4122-4$

Printed in Singapore. 
This book is dedicated to my wife, Shiow-Jen, my daughter Emy and my son Eden 
This page is intentionally left blank 


\section{PREFACE}

It is well recognized that AIDS is the most dangerous disease in the twenty first century threatening the survival of human beings. It has been estimated by WHO (World Health Organization) that by 1998 there are at least 40 million people world-wide who have contracted HIV and at least 12 million people have died of AIDS. From these statistics, obviously the most urgent challenging task is how to control the HIV epidemic. Because of the complexity and seriousness of the disease, for understanding the HIV epidemic and the HIV pathogenesis and for developing efficient strategies for controlling AIDS, mathematical models that take into account the dynamics of the HIV epidemic and the biology of the HIV pathogenesis are definitely needed. With this in mind, many mathematical models for the AIDS epidemic and for the HIV pathogenesis have been developed.

It appears that a large number of mathematical models in the literature are deterministic models in which the state variables are assumed as deterministic functions of time, ignoring completely randomness of the AIDS epidemic and the HIV pathogenesis. On the other hand, statisticians have developed statistical models to estimate the HIV prevalence and to project future AIDS cases, ignoring completely the dynamics of the HIV epidemic. There are several books published on deterministic models of the HIV epidemic and on statistical models; yet books on stochastic models of the AIDS epidemic and the HIV pathogenesis, especially state space models, remain to be published. The purpose of this book is therefore to present a systematic approach to stochastic models, statistical models and state space models for the AIDS epidemic and the HIV pathogenesis.

This book is organized into 9 chapters. In Chapter 1 , we will discuss and compare different modeling approaches and illustrate these approaches by using an example from the AIDS epidemic in homosexual populations. To provide some necessary background, in Chapter 2 we will present some basic concepts and basic stochastic processes related to the AIDS epidemic and the HIV pathogenesis. Most of these processes will be used in latter chapters for the development of stochastic models of the AIDS epidemic and the HIV pathogenesis. Having setup the basic background, in Chapter 3, I will proceed to illustrate how to develop stochastic models for the HIV epidemic and illustrate its applications. These are population-based transmission models of the HIV epidemic. In Chapter 4, I will review some basic approaches of statistical models and methods for estimating the HIV infection distribution and the HIV incubation distribution. Because the backcalculation method is a popular statistic model used widely by statisticians, in Chapter 5 , I will present and discuss the basic methodology and illustrate its applications. Having developed stochastic models and statistical models, in Chapter 6, I will unify them into state space models and illustrate its application. In Chapters 7-8, I will illustrate how to develop stochastic models of HIV pathogenesis in HIV-infected individuals. By 
using these stochastic models, in Chapter 9, I will then proceed to develop some state space models of HIV pathogenesis in HIV-infected individuals.

I originally compiled this book in the fall of 1993 for a seminar course for my students in the Department of Mathematical Sciences at the University of Memphis, Memphis, Tennessee, when I was giving a series of lectures on mathematical models of the AIDS epidemic. These lecture notes have then been up-dated and expanded to include state space models for the AIDS epidemic and stochastic models and state space models for the HIV pathogenesis in HIV-infected individuals. I have also presented some of these materials in a series of lectures in statistical workshops in Taiwan during the summers of 1996, 1997 and 1998. These workshops were organized by National Chung-Hsing University, Taichung, Taiwan (1996), FengChia University, Taichung, Taiwan (1997) and the National Chia-Tung University, Hsin-Chun, Taiwan (1998), and were supported by the National Science Council of the Republic of China, Taiwan. The materials of this book are intended for applied mathematicians, applied probability scientists, biostatisticians, biomedical researchers, AIDS researchers and medical professionals. As an unique feature of the book, I have dealt with in detail stochestic models and state space models of the AIDS epidemic and the HIV pathogenesis. Thus, the book may be used as a reference book for courses in epidemiology, in mathematical modeling and in stochastic models of biomedical systems. It may also be used as a text for seminar courses in biomedical systems and AIDS.

Most of the materials in this book were derived from my own research, and from joint research with my colleagues and friends Dr. R. Byers of CDC, Dr. Zhi-hua Xiang of Scirex Corporation, Dr. S. C. Tang of Cendant Corporation, Dr. S. R. Lee of Atlantic Search Group and Dr. Zheng-zheng Ye of University of Memphis. Most of these researchers were supported by grants from the National Institute of Allegy and Infectious Deseases/National Institutes of Health, Grant No. RO1 AI31869 and R21 AI31869. Without these supports, I would never have engaged in extensive research on AIDS.

I would like to express my sincere appreciation to Prof. Ying-Hen Hsieh of the National Chung-Hsin University, Professors Shen-Ming Lee and Cathy W. S. Chen, Dr. Nae-Sheng Wang and Dr. Mei-Jih Gee of the Feng-Chia University, and Prof. Jack C. Lee of the National Chiao-Tung University in Taiwan for organizing workshops in Taiwan and to the National Science Council of the Republic of China for its support. I want to thank my friends and former students Dr. S. C. Tang, Dr. S. R. Lee, Dr. Zhi-hua Xiang, Dr. Zheng-zheng Ye, Ms. W. H. Fan and Mr. Z. H. Zhu for their help in numerical computations and for drawing many 
of the figures in Chapters 3-9.

Finally I wish to thank Ms. Diane Mittelmeier of the Department of Mathematical Sciences at the University of Memphis for her help in compiling the Latex files, and also Dr. Sen Hu and Ms. T. Yugarani of the World Scientific Publishing Company for their assistance in the publication of my book.

Wai-Yuan Tan

February 2000 
This page is intentionally left blank 


\section{CONTENTS}

Preface vii

Chapter One: Introduction 1

1.1. The Role and Importance of Mathematical Models for AIDS 2

1.2. Different Modeling Approaches for Modeling the AIDS

Epidemic and the HIV Pathogenesis in HIV-infected Individuals 2

1.3. An Illustrative Example from the AIDS Epidemic 5

$\begin{array}{ll}\text { 1.4. The Scope of the Book } & 17\end{array}$

1.5. Complements and Exercises 20

1.6. References $\quad 20$

1.7. Appendices 27

Chapter Two: Some Basic Concepts and Stochastic Processes for Modeling the AIDS Epidemic and the HIV Pathogenesis

2.1. Stochastic Processes and Examples from AIDS 33

2.2. Markov Processes vs Non-Markovian Processes and Examples from AIDS

2.3. Binomial Distributions, Poisson Distributions and Multinomial Distributions

2.4. The Negative Binomial Distributions and the Delayed Negative Binomial Distributions

2.5. Some Stochastic Birth-Death Processes and Applications to AIDS

2.6. Some First Passage Times in AIDS and the HIV Incubation Distributions

2.7. Complements and Exercises $\quad 67$

$\begin{array}{ll}\text { 2.8. References } & 70\end{array}$

Chapter Three: Some Stochastic Transmission Models of the HIV Epidemic

3.1. Some Basic Procedures for Developing HIV Stochastic Models Homosexual Populations

3.3. Some Features of the Model via Monte Carlo Studies

3.5. Some Stochastic Staged Models of the HIV Epidemic in Homosexual Populations 
3.6. Extensions of Stochastic Models of HIV Epidemic to Models under Complex Situations

3.7. Some Applications of the Stochastic Models 123

3.8. Complements and Exercises 124

3.9. References 127

Chapter Four: Statistical Modeling of the HIV Epidemic

4.1. Some General Waiting-Time Distributions for the HIV Epidemic

4.2. The Infection Distribution and the Seroconversion Distribution

4.3. Some Statistical Methods for Estimating the HIV Seroconversion Distribution

4.4. Characterization of the HIV Infection Distribution and the Seroconversion Distribution

4.5. The Fourier Transform Method 163

4.6. The HIV Incubation Distribution 165

4.7. Some Statistical Methods for Estimating the HIV Incubation Distribution by Using Blood Transfusion Data 172

4.8. Characterization of the HIV Incubation Distribution

4.9. Effects of Risk Factors on the HIV Infection, the HIV Seroconversion and the HIV Incubation Distributions

4.10. Complements and Exercises

4.11. References

Chapter Five: The Backcalculation Method for the HIV Epidemic

5.1. Limitations and Assumptions of the Method

5.2. The Model and the Likelihood Function

5.3. Estimating the HIV Infection Distribution by Backcalculation

5.4. Estimating the HIV Incubation Distribution by Backcalculation

5.5. Estimating the Number of People at Risk for AIDS by Backcalculation

5.6. The Bayesian Method for Estimating the HIV Infection and the Incubation by Backcalculation

5.7. Short Term Projection of Future AIDS Cases by Backcalculation

5.8. Simultaneous Estimation of the HIV Infection Distribution and the HIV Incubation Distribution 
5.9. Complements and Exercises 231

5.10. References 232

Chapter Six: Some State Space Models of the HIV Epidemic and Applications

6.1. Some HIV Epidemic Models as Discrete-Time Linear State Space Models

6.2. Some General Theories for Discrete-Time Linear State Space Models

6.3. Estimation of HIV Prevalence and AIDS Cases in the San Francisco Homosexual Population

6.4. Some General Procedures for Estimating Simultaneously the Unknown Parameters and the State Variables by State Space Models

6.5. Simultaneous Estimation of the HIV Infection, the HIV Incubation and State Variables in the San Francisco Homosexual Population

6.6. Simultaneous Estimation of the HIV Infection, the HIV Incubation, the Immigration Rate, the Death Rate and the State Variables in the Swiss Homosexual Population

6.7. Complements and Exercises

6.8. References

6.9. Appendices

Chapter Seven: Some Stochastic Models of HIV Pathogenesis in HIV-Infected Individuals in the Absence of Anti-Viral Treatment

7.1. Some Biological Background and Observations

7.2. A Simple Stochastic Model of HIV Pathogenesis in HIV-Infected Individuals

7.3. Stochastic Models of HIV Pathogenesis under Complex Situations in HIV-Infected Individuals

7.4. Some Specific Features of HIV Pathogenesis via Monte Carlo Studies

7.5. Complements and Exercises 333

7.6. References

Chapter Eight: Stochastic Models of HIV Pathogenesis Under Treatment by Anti-Viral Drugs

8.1. Some Biological Background of Treatment by Anti-Retroviral Drugs 
8.2. Some Stochastic Models under Treatment by Anti-Retroviral Drugs in the Absence of Drug Resistance

8.3. Some Stochastic Models under Treatment by Anti-Retroviral Drugs in the Presence of Drug Resistance

8.4. Stochastic Models of HIV Pathogenesis under Treatment by a RT Inhibitor and Development of Drug Resistance

8.5. Stochastic Models of HIV Pathogenesis under Treatment by a Protease Inhibitor and Development of Drug Resistance

8.6. Some Features of Drug Resistance via Monte Carlo Studies

8.7. Complements and Exercises

8.8. References

Chapter Nine: Some State Space Models of HIV Pathogenesis in HIV-Infected Individuals

9.1. Some State Space Models of HIV Pathogenesis as Nonlinear State Space Models

9.2. Some General Theories of Continuous Time-Discrete Time Linear State Space Models

9.3. Estimation of the Numbers of Different Types of CD4(+) T Cells and Free HIV in Blood by State Space Models

9.4. Assessing Effects of Treatment by Anti-Retroviral Drugs by State Space Models

9.7. Appendix 


\section{ERRATA}

\section{STOCHASTIC MODELING OF AIDS EPIDEMIOLOGY AND HIV PATHOGENESIS}

\section{CHAPTER ONE \\ INTRODUCTION}

In Page 8 of Chapter 1, the first paragraph should be read as:

If $c(t)=c, q(t)=q$ and $\gamma(t)=\gamma$, then $\lambda(t)=c q-\gamma=\lambda$ is independent of $t$. ( This is the homogeneous model. ) In this case, the above solutions can be simplified to:

$$
\begin{gathered}
N(t)=N(0)\{[1-\psi(0)]+\psi(0) \exp (\lambda t)\}^{-\gamma / \lambda}, \\
I(t)=N(0) \frac{\psi(0) \exp (\lambda t)}{\{[1-\psi(0)]+\psi(0) \exp (\lambda t)\}^{1+(\gamma / \lambda)}} .
\end{gathered}
$$

Hence, if $\lambda(t)=\lambda$,

$$
S(t)=N(t)-I(t)=N(0) \frac{1-\psi(0)}{\{[1-\psi(0)]+\psi(0) \exp (\lambda t)\}^{1+(\gamma / \lambda)}} .
$$

The above results imply that the fraction $\psi(t)$ behaves along the logistic growth law whereas $I(t)$ behaves along the generalized log logistic growth law [80]. In the homogeneous case, the above results suggest that the progression of these numbers are determined by $\lambda=(c q-\gamma)=\gamma\left(R_{0}-1\right)$, where $R_{0}=c q / \gamma$ has been referred to as the reproductive number by Anderson et al [78]. This number may be interpreted as the average number an $I$ person will infect if he/she is put in a population of $S$ people. Notice that if $R_{0}>$ $1($ or $\lambda>0)$, then $\lim _{t \rightarrow \infty} I(t)=0, \lim _{t \rightarrow \infty} \psi(t)=1, \lim _{t \rightarrow \infty} I(t)=0$ and $\lim _{t \rightarrow \infty} S(t)=\lim _{t \rightarrow \infty} N(t)=0$. On the other hand, if $R_{0} \leq 1($ or $\lambda \leq 0)$, then $\lim _{t \rightarrow \infty} S(t)=\lim _{t \rightarrow \infty} N(t)=N(0)\{1-\psi(0)\}^{\gamma /|\lambda|}$ which is a positive number. 
In page 14 of Chapter 1, equation (1.29) should be changed to:

$$
\begin{aligned}
P\left(t_{j-1}, t_{j}\right) & =\int_{t_{j-1}}^{t_{j}} \int_{t_{0}}^{t} f\left(t_{0}, x\right) g(x, t) d x d t \\
& =\left\{\int_{t_{0}}^{t_{j}}-\int_{t_{0}}^{t_{j-1}}\right\} \int_{t_{0}}^{t} f\left(t_{0}, x\right) g(x, t) d x d t \\
& =\int_{t_{0}}^{t_{j}} f\left(t_{0}, x\right)\left\{G\left(x, t_{j}\right)-G\left(x, t_{j-1}\right) d x\right.
\end{aligned}
$$

